\title{
Temporal and spatial factors in the fading of diffuse visual images
}

\author{
BARRY KIRKWOOD \\ UNIVERSITY OF OTAGO, New Zealand
}

Pattern vision breaks down after a period of inspecting a diffusely contoured dark figure upon a ground of uniform brightness and texture. When the field is illuminated with flickering light it is found that the persistence of pattern vision is inversely related to flicker frequency up to the region of fusion. At low flicker frequencies complex figures persist longer than less complex ones of equivalent area, but the reverse applies at frequencies above fusion. These findings are consistent with data obtained from experiments using stabilized retinal images.

It is now well established that a marked deterioration in visual efficiency occurs when an observer inspects a uniformly illuminated homogeneously textured field or Ganzfeld (Cohen, 1957). Even areas of inhomogeneous hue or brightness introduced into an otherwise uniform field are perceptually unstable and seem to fade into their background after a time (Guilford, 1927; Cohen, 1958; Kirkwood 1967). These effects appear consistent with findings from work with stabilized retinal images. Under these conditions, if the target consists of a black bar upon a bright field, then light from the field appears to invade the dark area until the whole bar disappears and the field appears uniformly illuminated. For a given accuracy of stabilization the time of disappearance of the image of a black bar upon a bright field is directly related to bar wid th, fine lines disappearing rapidly while wide bars take longer to fade (Riggs et al, 1953, Ditchburn \& Fender, 1955). Similar effects have been reported using bar gratings and with fringe patterns (Ditchburn \& Pritchard, 1956).

To date there is a dearth of equivalent data on the effects of systematically introducing inhomogeneities of luminance into a Ganzfeld. Luminance differentials can occur in both space and time. In the present study the spatial case is investigated by systematically varying both the area and configuration of a dark region upon a surface which is of otherwise homogeneous brightness and texture. Temporal discontinuity is obtained by illuminating the field with intermittent light, the flicker rate extending from a low frequency to a rate above fusion, where illumination is subjectively continuous.

\section{METHOD}

Homogeneous visual stimulation was obtained by means of eye caps fashioned from half table-tennis balls shaped to fit snugly around the orbit of the eye. The S lay supine and looked directly upward at the light source, an E.M.I. Type 4 electronic stroboscope covered by an opal glass diffusing screen. The stroboscope was modified to give pulse rates ranging from 1.2 to $72 \mathrm{cps}$. Pulse rate and light intensity were continuously monitored by means of a phototransistor detector and a Tektronix 502A oscilloscope. Pulse height and shape were uniform within $\pm 1 \%$ at the frequencies used. The pulses emitted had a rise time of $20 \mu \mathrm{sec}$ followed by an ogival decay falling to half amplitude at $800 \mu \mathrm{sec}$. At 72 cps flicker, well above flicker fusion, the luminance of the inner surface of a plain eye cap placed at S's position was equivalent to $0.9 \mathrm{ft}-\mathrm{L}$ measured with an S.E.I. photometer. All observations were carried out in a darkened room.

Seven eye caps were constructed with patterns described upon their outer surfaces with two coats of matt black acrylic paint. The patterns, see Fig. 1, were: (A) single vertical line, width $1 \mathrm{~mm}$ subtending a visual angle of 3 deg approx.; (B) single vertical bar, width $5 \mathrm{~mm}$; (C) two parallel vertical bars each $2.5 \mathrm{~mm}$ wide separated $2.5 \mathrm{~mm}$; (D) vertical division of the field into opaque and translucent halves; (E) vertical stripes $2.5 \mathrm{~mm}$ wide separated $2.5 \mathrm{~mm}$ covering entire field; (F) lattice of horizontal and vertical lines $1 \mathrm{~mm}$ wide; (G) opaque field except for a central circular area, diameter $1 \mathrm{~cm}$, vertically bisected by a $1 \mathrm{~mm}$ line.
Targets $B$ and $C$ have equal opaque areas as have $D, E$, and F, these latter having half the area of the hemisphere opaque. Equating opaque areas in this way permits evaluation of the effect of configuration largely unconfounded by variations in opaque area, although the physical contrast of the smaller dark regions is also reduced relative to the large opaque areas due to the scattering of light within the cap. Pattern F, the lattice, and G the short line in the central field were designed to investigate differential fading in the foveal and peripheral regions.

The Ss were two young adults in good physical health with no readily detectable visual defects. The left eye was used for all observations, the right being occluded by a patch. The $S$ was instructed to report verbally when a uniform gray field was perceived, except for figure $G$ where the criterion was disappearance of the central line. At the beginning of each trial the eye was closed for $10 \mathrm{sec}, S$ then opened his eye, the stroboscope started and $E$ measured the time between eye opening and the report of contour fading. Seven flicker rates were used: 1.2, 5.4, $9.5,13.5,22,38$, and $72 \mathrm{cps}$, run in random order. A total of 16 observations were made with each pattern at each flicker rate, except for pattern A where 24 observations were made under each condition.

At the end of the main experiment the critical flicker fusion frequency (cff) was determined by the method of ascending and descending series. In this part of the experiment the eye was covered by a plain translucent eye cap. The mean cff was 39.06 cps, (S.E.M $=0.865$ ) on the basis of 16 observations.

\section{RESULTS}

Similar results were obtained from both Ss. The data have therefore been combined and the averaged results shown in Figs. 2 and 3. All persistence times refer to the initial disappearance of contour following the start of each experimental run; loss of contour vision is usually brief and can be restored by $\mathbf{S}$ blinking or making gross eye movements (Kirkwood, 1967).

Figures 2 and 3 show that for all target patterns the mean persistence time is inversely related to flicker frequency up to the cff region. (There is a linear correlation coefficient of -0.89 $(p=0.01)$ between persistence time and the square root of flicker frequency over the range 1.2 to $38 \mathrm{cps}$, taken over all observations.) Above fusion, over the limited range studied, mean persistence times do not show any significant change with increasing flicker frequency, except for pattern $D$ which shows significantly longer persistence time $(p=0.01)$ at $72 \mathrm{cps}$ than it does at 38 cps. This difference may be a result of fortuitous
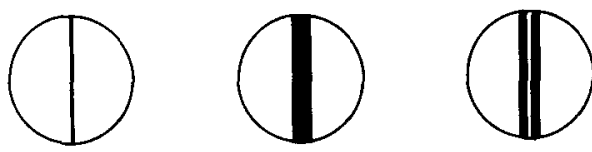

A

B
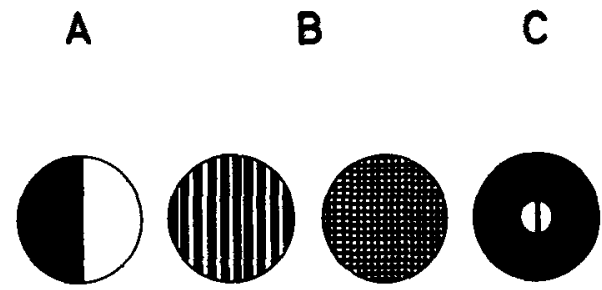

D

E

$\mathbf{F}$

G

Fig. 1. Target patterns used in the experiman. 


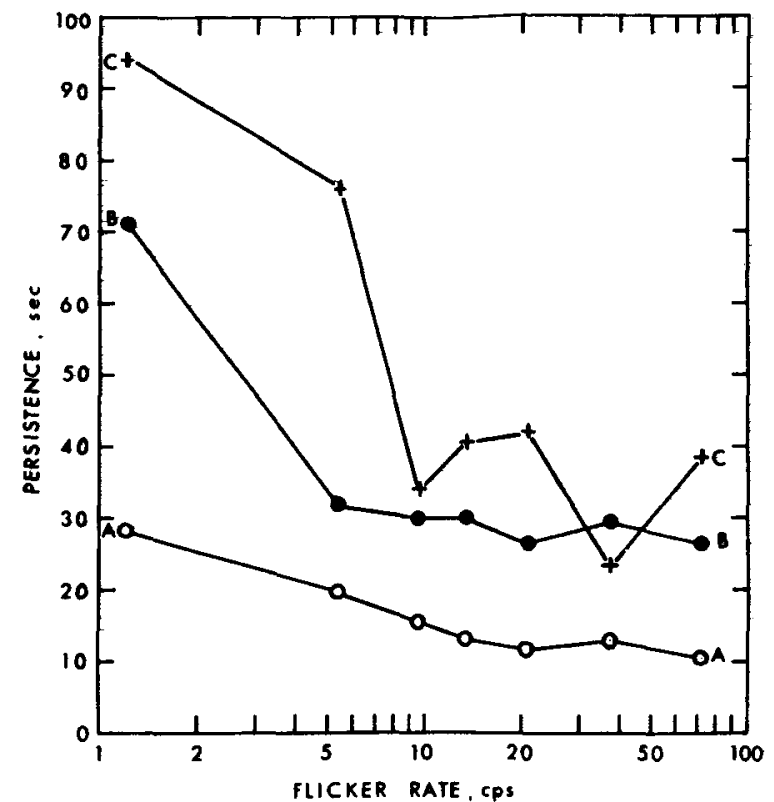

Fig. 2. Relationship between mean persistence time and flicker rate for patterns A, B, C. Standard deviations average 0.38 of mean persistence times shown.

sampling from the large number of observations taken. The mean persistence times at any flicker frequency do not seem to have any simple relationship with the judged brightness of the flashes which appeared to peak around $1.2,9.5$ and $72 \mathrm{cps}$.

The targets studied vary with respect to: (1) total opaque area; (2) topological complexity; (3) width of individual pattern elements. A positive relationship exists between the gross opaque area of a figure and persistence of contour perception at all flicker rates used. Target $A$, a $1 \mathrm{~mm}$ line shows least persistence, while one or another of the figures that occlude half the field show greatest persistence at any flicker rate. Maintenance of contour perception is not, however, a simple function of target dark area. Target $C$, for example, consisting of two $2.5 \mathrm{~mm}$ bars is seen for a significantly longer $(p=<.01)$ period than target $B$, a single bar of $5 \mathrm{~mm}$ width having an equal opaque area. Using Kendall's partial rank correlation technique it was found that both complexity of figure and gross opaque area showed similar positive correlations (tau $=0.34,0.46$, respectively) with persistence time after their mutual correlation had been partialled out.

Figures 2 and 3 show that the relative persistence times of certain targets, notably D, E, and F change over the range of flicker frequencies. Rank order correlation coefficients were calculated between the parameters of persistence time, opaque area, topological complexity and width of individual pattern elements at 1.2 and $72 \mathrm{cps}$ flicker. The results are shown in Table 1. None of obtained coefficients differed significantly from zero, except for complexity of figure which showed a positive correlation (rho $=0.66$ ) approaching the $p=0.05$ level with persistence under slow flicker and had a weak negative correlation (rho $=-0.38$ ) at $72 \mathrm{cps}$ flicker. This implies that the presence of a relatively large number of well defined contours in the visual field may aid pattern perception when illumination is intermittent, but have negligible, or possibly adverse effect under conditions of continuous illumination.

Reports were obtained from Ss in this experiment as well as from Ss in a less formal preliminary investigation as to any area of the field which showed differential loss of contour vision. The lattice pattern $F$ was useful in this respect. There was consistent report that vision in the central part of the field seemed most prone to fading, the loss of contour seeming to spread out from the center to the periphery.

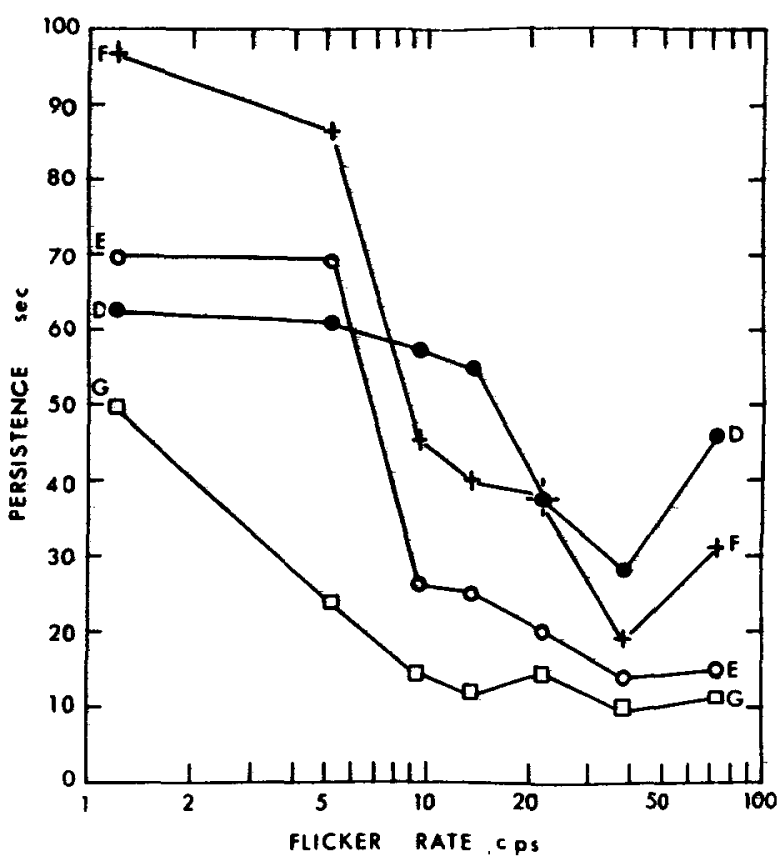

Fig. 3. Relationship between mean persistence time and flicker rate for patterns, D, E, F, G. Standard deviations average 0.38 of mean persistence times shown.

\section{DISCUSSION}

The data have shown that contour perception can break down without stabilization of the retinal image under photopic conditions even when the inspection field is so strongly patterned that Ganzfeld conditions cannot be said to obtain, a finding in accord with earlier work at mesopic luminances by Guilford (1927), and Fry and Robertson (1935). There is a positive relationship between the persistence of pattern vision and the area of dark figures upon an otherwise homogeneously bright field, up to the limiting case of $50 \%$ opaque area used in this study. Persistence of pattern vision is also found to be better at low flicker frequencies than it is when illumination is subjectively continuous.

These results are in many respects similar to data obtained from experiments with stabilized retinal images, as might be expected from the evidence that the reports of fading of images under conditions similar to those used in this experiment are invariably associated with periods of reduced eye movement (Kirkwood, 1967). Riggs et al (1953) reported that images of fine lines fade more rapidly than those of thick bars when stabilized on the retina. Cornsweet (1956) found that the persistence of a stabilized retinal image was inversely proportional to the frequency of flickering illumination of the field over a range of 0.9 to $9.6 \mathrm{cps}$, a result consistent with the findings reported above. Evans (1965) found that there was a negative relationship between figural complexity and persistence of stabilized images under continuous illumination. There is some evidence of a similar trend in the data reported here when flicker frequencies above fusion are used, although the correlation coefficient obtained only approaches the

Table 1

Rank Correlations between Experiment Parameters at Low and High Flicker Rates

\begin{tabular}{llllll} 
& \multicolumn{2}{c}{ Time } & \multicolumn{1}{c}{ Area } & Complexity \\
\hline Area of Figure & 0.55 & $(0.64)$ & & & \\
Complexity of Figure & $0.66(-0.38)$ & 0.71 & $(0.71)$ & & \\
Width of Fig. Elements & $0.31(0.28)$ & 0.32 & $(0.32)$ & $-0.20 \quad(-0.20)$ \\
\hline
\end{tabular}

Spearman Rank Correlation Coefficients. Figures in brackets refer to observations with $72 \mathrm{cps}$ flicker, figures outside brackets to $1.2 \mathrm{cps}$ ficker. $N=7, p .05=0.714$. 
$p=0.05$ level if a one-tailed test is used, and the relationship must therefore be considered tenuous.

Does a similar adaptational mechanism underlie the deterioration in pattern vision observed both in this experiment and with retinal image stabilization? The conditions differ in two obvious respects: Firstly, there is no stabilization of images upon the retina when the $O$ inspects the inner surface of the eye cap. Secondly, while care is usually taken to ensure precise accommodation of the focus of the eye to the test figure in stabilized retinal image experiments, the eye caps used in this study are so close to the eye that the image is never sharply resolved, and the phenomenal appearance of a contour drawn upon the cap is of a blurred edge shading gradually into the background. This reduces the effect of small eye movements as the rate of change of luminance is less across a blurred contour than it is across a sharp one. There are also data from Enoch (1958) and Thomas and Kovar (1965) showing that perceived figure-ground contrast is reduced as the sharpness of the boundary separating figure from ground is decreased.

The results of this experiment can thus be accounted for in terms of lateral inhibitory interactions such as those observed in Limulus (Ratliff et al, 1963) and suggested for human vision (von Békésy, 1960). As the differentials in excitation between adjacent neural elements in the region of a diffuse contour would be small and relatively little change would occur as a result of micronystagmus the model would predict that after a time adaptation would be complete and all units would reach a uniform level of activity resulting in a loss of response to contour, as is the case in this experiment. As this adaptation takes time to occur it would also be expected that figure-ground differentials would be better maintained at low flicker frequencies. This prediction is sustained by the results of this experiment and also by Bittini et al (1960) who reported enhanced contrast of indefinitely contoured objects with intermittent illumination. An additional factor that would aid contour perception under flickering light is the reported increase in saccade rate induced at flicker frequencies in the region of 1 to $3 \mathrm{cps}$ (West \& Boyce, 1968). Independent of the correctness of this explanation, the data clearly show that even large diffusely contoured figures on a ground of uniform brightness and texture can be perceptually unstable.

\section{REFERENCES}

BITTINI, M., ERCOLES, A. M., FIORENTINI, A., RONCHI, L., \& FRANCHIA, G. T. Enhanced contrast of an indefinitely contoured object by movement or intermittent illumination. Pubbl. Inst. naz. Office, 1960, $15,62-84$.

COHEN, W. Spatial and textural characteristics of the Ganzfeld. Amer. J. Psychol., 1957, 70, 403-410.

COHEN, W. Color perception in the chromatic Ganzfeld. Amer. J. Psychol, $1958,71,390-394$.

CORNSWEET, T. N. Determination of the stimuli for involuntary drifts and saccadic eye movements. J. Opt. Soc. Amer., 1956, 46, 987-993.

DITCHBURN, R. W., \& FENDER, D. H. The stabilized retinal image. Optica Acta, 1955, 2, 128-133.

DITCHBURN, R. W., \& PRITCHARD, R. M. Stabilized interference fringes on the retina. Nature, 1956, 177, 434.

ENOCH, J. M. Summated response of the retina to light entering different parts of the pupil. J. Opt. Soc. Amer., 1958, 48, 392-405.

EVANS, C. R. Some studies of pattern perception using a stabilized retinal image. Brit. J. Psychol., 1965, 56, 121-134.

FRY, G. A., \& ROBERTSON, V: M. The physiologic basis of the periodic merging of area into background. Amer. J. Psychol., 1935, 47, 644-655.

GUILFORD, J. P. Fluctuations of attention with weak visual stimuli. Amer. J. Psychol., 1927, 38, 534-583.

KIRKWOOD, B. Relationship between the electroencephalogram, eye movements, and fluctuations of visual perception. Psychon. Sci., 1967, 7, 417-418.

RATLIFF, F., HARTLINE, H. K., \& MILLER, W. H. Spatial and temporal aspects of retinal inhibitory interaction. J. Opt. Soc. Amer., 1963, 53, $110-120$.

RIGGS, L. A., RATLIFF, F., CORNSWEET, J. C., \& CORNSWEET, T. N. The disappearance of steadily fixated visual test objects. J. Opt. Soc. Amer., 1953, 43, 495-501.

THOMAS, J. P., \& KOVAR, C. W. The effect of contour sharpness on perceived brightness. Vision Res., 1965, 5, 559-564.

von BÉKÉSY, G. Neural inhibitory units of the eye and skin. Quantitative description of contrast phenomena. J. Opt. Soc. Amer., 1960, 50, 1060-1070.

WEST, D. C., \& BOYCE, P. R. Effect of flicker on eye movements. Vision Res., 1968, 8, 171-192.

NOTE

1. Address: Department of Psychology, University of Otago, P. O. Box 56, Dunedin, N.Z.

(Accepted for publication on April 16, 1968.) 